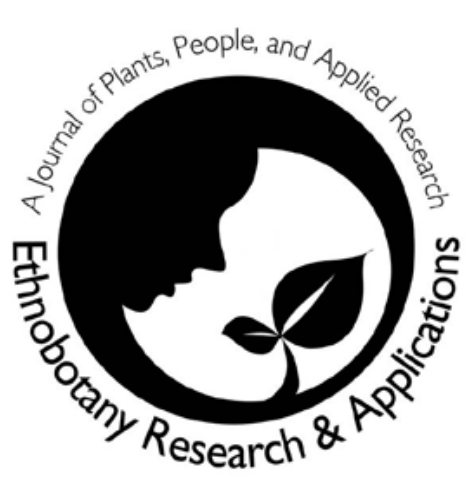

\title{
Ethnobotanical Study of the Medicinal Plants Known by Men in Ambalabe, Madagascar
}

\author{
Aina D. Rabearivony, Alyse R. Kuhlman, Zo Lalaina \\ Razafiarison, Fidèle Raharimalala, Fortunat Rakotoarivony, \\ Tabita Randrianarivony, Nivo Rakotoarivelo, Armand \\ Randrianasolo, and Rainer W. Bussmann
}

\section{Research}

\begin{abstract}
Madagascar has high biodiversity and endemism that are threatened by growing human populations and climate change. Species loss has potential impacts on traditional knowledge and community health. The aim of this project was to identify medicinal plants known and used by men in the Commune of Ambalabe sourced from the Vohibe Forest in eastern Madagascar. Interviews were conducted that resulted in collection of 137 plant species used by men to treat illnesses, $35 \%$ of which are endemic to Madagascar. Twelve tree species were shown to have the highest Use Index among the men in Ambalabe: Noronhia gracilipes H.Perrier, Xylopia humblotiana Baill., Fenerivia ghesquiereana (Cavaco \& Keraudren) R.M.K.Saunders, Phyllarthron bojeranum DC., Mauloutchia humblotii (H.Perrier) Capuron, Aphloia theiformis (Vahl) Benn., Dillenia triquetra (Rottb.) Gilg, Chrysophyllum boivinianum (Pierre) Baehni, Oncostemum boivinianum H.Perrier, Peponidium humbertianum (Cavaco) Razafim., Lantz \& B.Bremer, Eugenia goviala H.Perrier, and Macaranga alnifolia Baker.
\end{abstract}

\section{Background}

Madagascar, like many tropical countries, is confronted with biodiversity loss and conservation problems as a result of the continued destruction of natural habitats (Gade 1996, Gallegos 1997, Goodman \& Patterson 1997, Green \& Sussmann 1990, Mittermeier et al. 1994). This problem is usually caused by bush fires, fast clearing of vegetation for agricultural expansion, and forest exploitation and is exacerbated by climate change and high levels of poverty (Busch et al. 2012). These factors seriously affect the areas of primary forest in Madagascar and thus lead to a dramatic decline in the number of medicinal plant species available (Novy 1997) which in turn may lead to the de- cline in ecological and cultural knowledge regarding traditional healing (Lyon \& Hardesty 2005). Malagasy rural people are highly dependent on medicinal plants for their healthcare needs, particularly to treat infectious disease (Randrianarivelojosia et al. 2003). Socio-economically, the practice of traditional medicine helps to meet certain needs of the local population (Rajerison 1999). Economically, local residents have difficulty accessing modern drugs because of their high costs. The use of traditional medicine is cost effective as harvesting plants for therapeutic use often costs significantly less than buying pharmaceuticals. Furthermore, people turn to traditional remedies as they believe they have little to no side effects.

\section{Correspondence}

Aina D. Rabearivony, Zo Lalaina Razafiarison, Fidèle Raharimalala, Department of Plant Biology and Ecology, Faculty of Science, University of Antananarivo, BP 566, Antananarivo 101, MADAGASCAR. aina_rabearivony@yahoo.fr

Alyse R. Kuhlman, Armand Randrianasolo, Rainer W. Bussmann, William L. Brown Center, Missouri Botanical Garden, P.O. Box 299, Saint Louis, Missouri 63166-0299, U.S.A. alyse.kuhlman@mobot.org, armand.randrianasolo@mobot.org, rainer.bussmann@mobot.org

Fortunat Rakotoarivony, Tabita Randrianarivony, Nivo Rakotoarivelo, Missouri Botanical Garden, Madagascar Research and Conservation Program, BP 3391, Antananarivo 101, MADAGASCAR. fortunat.rakotoarivony@ mobot-mg.org, tabita.randrianarivony@mobot-mg.org, nivo.rakotoarivelo@mobot-mg.org

Ethnobotany Research \& Applications 14:123-138 (2015)

Published: 25 January 2015 
This research contributes to the understanding of the socio-economic importance of these plants in the study area and works to better identify factors involved in the degradation and therefore conservation measures. This study was designed to document the forest species used as medicine by men, through self medication as well as by traditional healers. Our focus was on the response of men, while a similar study conducted at the same time focused on the knowledge and utilization of medicinal plants by the women of Ambalabe. We separate these two studies for both cultural and scientific reasons, and in the future we will compare the two bodies of knowledge. Culturally, we were sensitive to the community's desire to keep data separate so gender plant preference was not confused or lumped together. Scientifically, we were interested in analyzing the data separately to add to the growing body of gendered use studies (Ayantunde et al. 2008, Bussmann \& Glenn 2010, Camou-Guerrero et al. 2008, Wayland 2011). Our hypothesis was that men in the community have specialized plant knowledge pertaining to only male health concerns.

\section{Ambalabe community}

Vohibe Forest is located within the rural municipality of Ambalabe, District Vatomandry, Atsinanana Region, and is the primary natural resource for this community. The community is predominately Betsmisaraka, which is characterized by large families with their respective leaders as well as the tangalamena, who are generally the eldest males and considered community spiritual leaders and close to the ancestor spirits. According to the census conducted in 2010 by the community, the population of the rural municipality of Ambalabe consists of 9036 inhabitants: 4252 men and 4784 women.

\section{Access to markets and healthcare}

Ambalabe is extremely isolated from the bustling marketplace in Vatomandry, the closest port town on the Indian Ocean. A road and bridge once connected Ambalabe to Vatomandry, but extreme weather destroyed the infrastructure in the 1970s. The community has been working to rebuild the road, but funding is problematic. The isolation of Ambalabe from other communities makes markets and healthcare options less accessible. Ambalabe Commune has a Center of Basic Health Level II (CSB II) located in the largest fokontany, or village, also named Ambalabe. However, the center cannot meet the demand for medical care of the population of all villages. Therefore, only people with serious illnesses consult the doctor, which is why the population often seeks traditional plantbased medicine instead of making use of modern medicine. Traditional healers still play important roles because often they have demonstrated the efficacy of certain plants in treating many diseases (Andriamaholy 1994). Data collected in 2006 show the CSB II found that patients were seen primarily for malaria (60\%) and diarrhea (30\%), with the remaining $10 \%$ seen for various diseases related to parasite infections.

\section{Livelihood and economy}

Shifting cultivation (tavy) is a way of life for the Betsimisaraka (Quansah 1988). A plot of cleared natural forest is cultivated for one or two years, then left fallow for three to seven years before being cultivated again. In this period, pioneer species like Psiadia altissima (DC.) Drake, Trema orientalis (L.) Blume, Aframomum angustifolium (Sonn.) K.Schum., Lantana camara L., and Rubus mollucanus L. are monitored by the farmers as these species are considered indicators of soil fertility, and their growth signals that the land is suitable for cultivation. Farmers use watersheds for rice cultivation, with or without other food crops such as Manihot esculenta Crantz, Ipomoea batatas (L.) Lam., Musa spp., Colocasia antiquorum Schott, and Saccharum officinarum L. Subsistence agriculture is the primary occupation in eastern Madagascar, but daily living needs are supplemented by forest products (Byg \& Balslev 2001). Vohibe Forest provides firewood, timber, medicinal plants, and edible plants to the community.

\section{Methods}

\section{Study site}

Vohibe Forest is 3117 ha of low altitude moist evergreen forest and part of the Ankeniheny-Zahamena forest corridor, located in the northwestern part of the District of Vatomandry, Madagascar, between 48 $32^{\prime}-48^{\circ} 35^{\prime} \mathrm{E}$ and $19^{\circ} 06^{\prime}-19^{\circ} 10^{\prime} \mathrm{S}$ (Figure 1).

The topography alternates between valleys and mountains at altitudes of 400-1008 m (Rakotoarivony \& Rasoavity 2007). The study site is underlain with metamorphic rocks rich in hornblende, garnet, and graphite (Besairie \& Collignon 1972). The climate is tropical and humid with average annual rainfall at $1773 \mathrm{~mm}$ (Morat 1973). There are two seasons experienced in Vohibe Forest, the hot and humid season lasting from November to April (average temperature of $26.7^{\circ} \mathrm{C}$ ) and the cool season from May to October (the coolest month with an average temperature of $20.85^{\circ} \mathrm{C}$ ).

Vohibe Forest transitions between the low altitude (400$800 \mathrm{~m}$ ) dense evergreen forest characterized by a canopy of 20-30 m and the medium altitude forest (800-1008 $\mathrm{m}$ ) with canopies of $17 \mathrm{~m}$ high (Faramalala \& Rajeriarison 1999). Botanical inventories of Vohibe Forest have identified 672 species distributed in 126 families and 327 genera with a specific endemic rate of $76 \%$ (Rakotoarivelo et al. 2013). Primatologists have documented four families of lemurs within Vohibe Forest: the Indridae, the Lemuridae, the Cheirogaleidae, and the Daubentoniidae, 


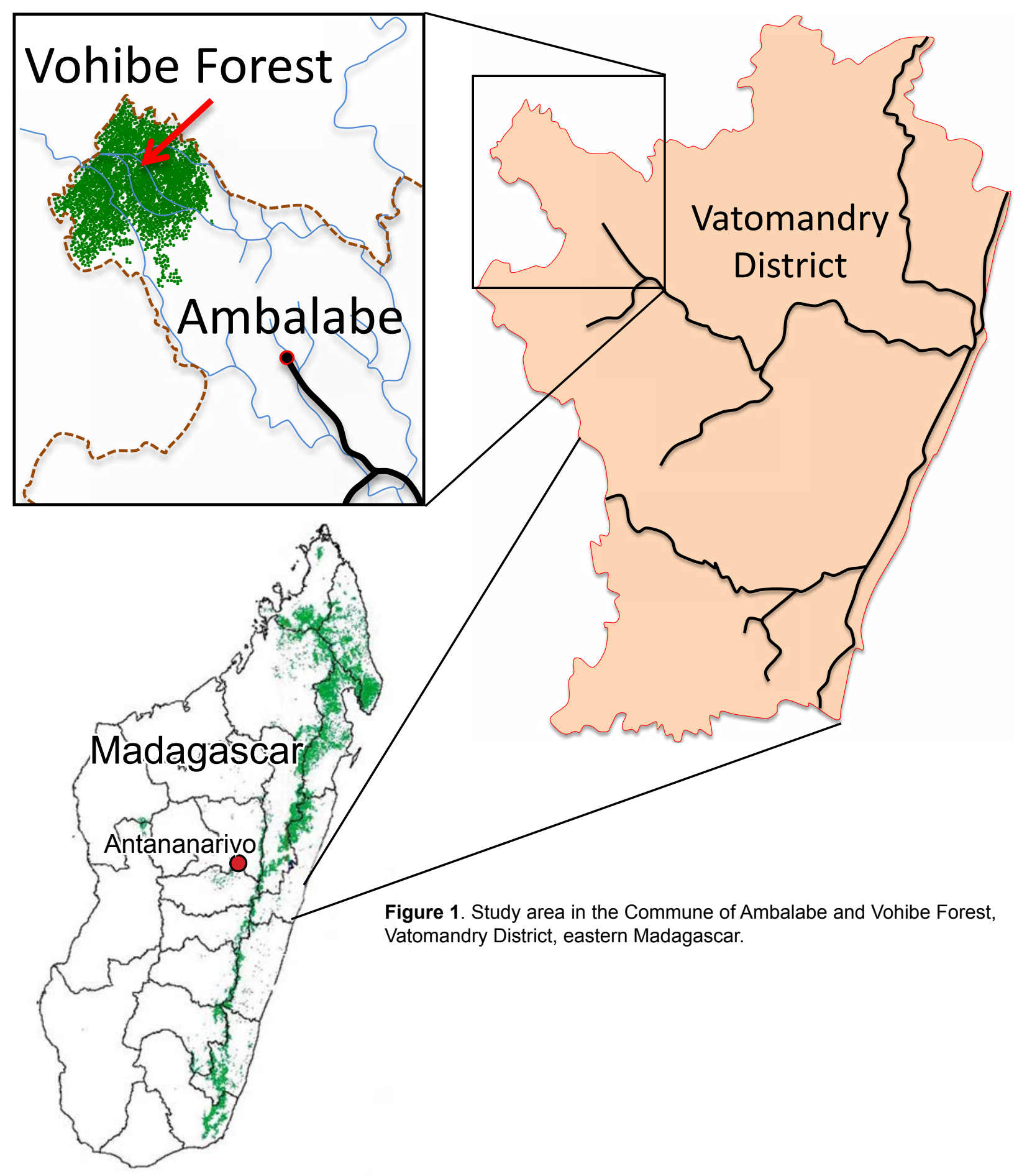


distributed across 10 species (Rakotoarivony \& Rasoavity 2007).

\section{Ethnobotanical surveys}

Ambalabe community has participated as a communitybased conservation site operated by the Missouri Botanical Garden since 2005. Collaborating with local staff to facilitate our ethnobotanical survey expedited the process of identifying potential informants and establishing interview schedules. Initial community visits were made in November to December 2008 and were devoted to making contact with the leaders of the village including the tangalamena, traditional leaders, and administrative heads of the municipality. Community meetings were widely advertised by our local collaborators before we arrived and were held in a community space. The preliminary phase was to familiarize ourselves with the residents and obtain a clear picture of the knowledge of medicinal plants by men and use of forest products in the life of the local population. A simultaneous study of the use of medicinal plants known and used by women was conducted by a female researcher. The results of that study are still pending.

The second field visit lasted 25 days in January-February 2009. Surveys were conducted with traditional healers, knowledgeable men, and the medical personnel of CSB II. Local guides facilitated introductions and helped identify traditional healers and those willing to share information. Informed consent was given by tribal leaders, government officials, and individuals prior to interviews. Information documented included species used, parts used, methods of collecting, locations of harvest, and diseases treated. Interviews were conducted in Malagasy by the first author, a native speaker, with dialect translation help by the local guide. In total, 177 men were interviewed from 18 villages in Ambalabe Commune. The ages ranged from 16 to 70 years of age (Figure 2). Participants listed occupations such as farmers, nurserymen, and government administrators.

Two modes of inquiry were employed: group interviews held in lapa, outdoor community spaces, and individual interviews with available men who were willing to share information. The surveys were conducted in the form of semi-direct questions with semi-open groups (Martin 1995). This approach allowed for flexibility in the conversation and open exchange with the informants. Interviews were conducted using two approaches: through illness (description of symptoms and designation of the plants used) or through the plant name (matching plant names to the therapeutic indications). Following the interviews, vouchers were collected both with the guides and with the local people in the areas of harvest. Plants were identified by their common name by the guides and local people. Vouchers were deposited at the Missouri Botanical Garden (MBG), the Herbarium of Parc de Tsimbazaza (TAN), and the Herbarium at the Centre National de la Recherche Appliquée au Developement Rural (TEF).

\section{Quantitative analysis}

To evaluate the species most used by the population, the Use Index (I\%) of each species was calculated using the formula (Lance et al. 1994):

$$
\mathrm{l} \%=\mathrm{n} / \mathrm{N} \times 100
$$

where $1 \%$ is the percentage index of use, $\mathrm{n}$ is the number of people citing the species, and $\mathrm{N}$ is the total number of people surveyed. If the value of $1 \%$ is between 60 and $100 \%$, the species is well known; if $1 \%$ is between 30 and

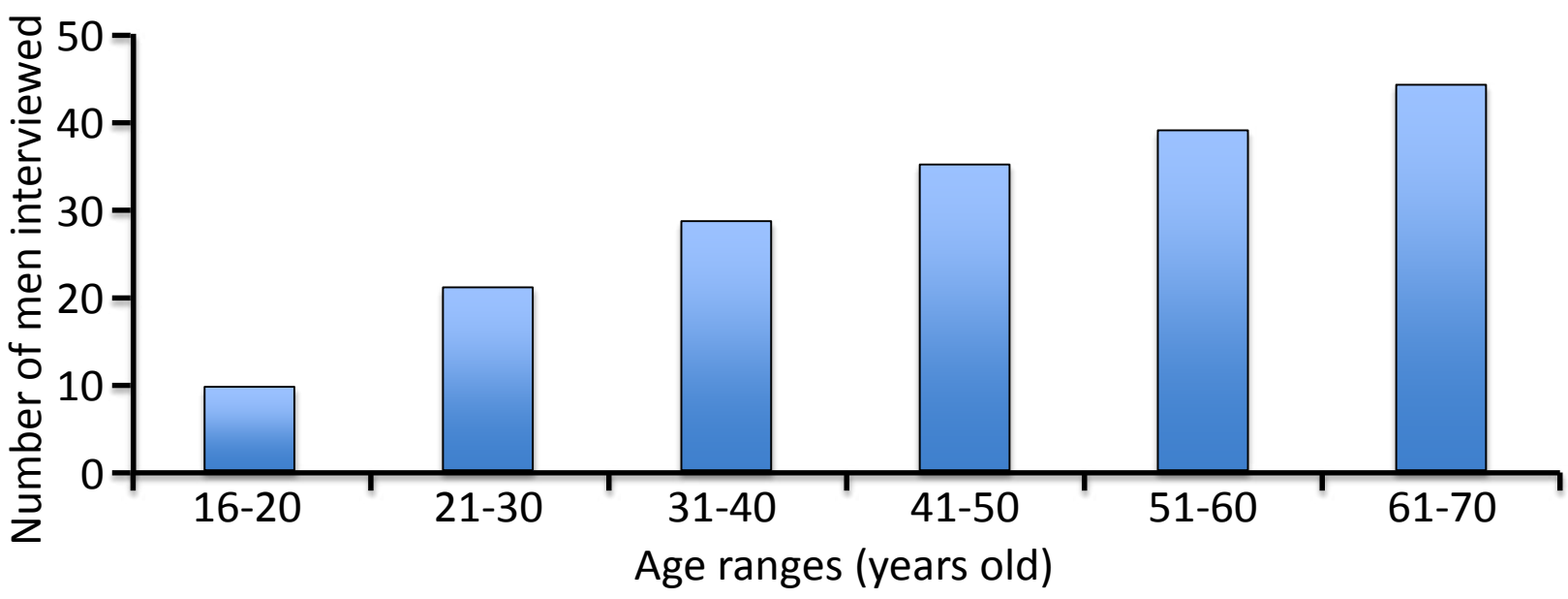

Figure 2. Men from the Commune of Ambalabe in eastern Madagascar interviewed about medicinal plants sourced from the Vohibe Forest. 


\section{Rabearivony et al. - Ethnobotanical study of the medicinal plants known by men in Ambalabe, Madagascar}

$60 \%$, the species is moderately known; if $1 \%$ is less than $30 \%$, the species is little known.

\section{Results}

The ethnobotanical survey revealed 137 forest and weedy species with therapeutic uses known by the men of Ambalabe Commune (Table 1). They belong to 69 families dis- tributed in 120 genera. Thirty-five percent of these species are endemic to Madagascar (MadCat 2012).

In the study area, almost all the different plant parts (leaves, stem, root, and fruit) are used for healing. Leaves have the highest percentage of use $(68 \%)$, followed by bark $(10 \%)$ and stems (9\%). Four methods of collecting

Table 1. Medicinal plants sourced from Vohibe Forest known by men of Ambalabe, Madagascar. Part used: B. Bark, E. Entire plant, F. Fruit, L. Leaf, R. Root, RF. Ripe fruit, and S. Stem. Distribution: E. Endemic to Madagascar, I. Indigenous or native to Madagascar,C. Comoros, M. Mascarene Islands, S. Seychelles, Af. Africa, Cu. Cultivated in Madagascar, Na. Naturalized in Madagascar, As. Asia, Au. Australasia, N. Neotropical, and NE. Not endemic or native to region.

\begin{tabular}{|c|c|c|c|c|}
\hline Scientific Names & Vernacular Names & $\begin{array}{l}\text { Part } \\
\text { used }\end{array}$ & Disease/Use & Distribution \\
\hline \multicolumn{5}{|l|}{ AMARANTHACEAE } \\
\hline Amaranthus sp. & Anampatsa & $S$ & Bilharzia & \\
\hline $\begin{array}{l}\text { Dysphania ambrosioides (L.) } \\
\text { Mosyakin \& Clemants }\end{array}$ & Taimborintsiloza & $L$ & Intestinal parasites & $\mathrm{Na}$ \\
\hline \multicolumn{5}{|l|}{ ANACARDIACEAE } \\
\hline $\begin{array}{l}\text { Abrahamia nitida (Engl.) } \\
\text { Randrian. \& Lowry }\end{array}$ & Sefana & $L$ & $\begin{array}{l}\text { Abdominal pain, urine retention, } \\
\text { injury }\end{array}$ & $E$ \\
\hline \multirow[t]{2}{*}{ Mangifera indica L. } & \multirow[t]{2}{*}{ Manga } & $L$ & Dental cavity & \multirow[t]{2}{*}{$\mathrm{Cu}$} \\
\hline & & $\mathrm{B}$ & Hemorrhoids, diarrhea & \\
\hline \multirow[t]{2}{*}{ Sorindeia madagascariensis DC. } & \multirow[t]{2}{*}{ Voasirindrina } & $L$ & $\begin{array}{l}\text { Cancer, stomach pain, urine } \\
\text { retention }\end{array}$ & \multirow[t]{2}{*}{ C, I, Af } \\
\hline & & $R$ & Back pain & \\
\hline \multicolumn{5}{|l|}{ ANNONACEAE } \\
\hline $\begin{array}{l}\text { Fenerivia ghesquiereana (Cavaco } \\
\text { \& Keraudren) R.M.K.Saunders }\end{array}$ & Ambavy & B & Gonorrhea, sexual problems & $E$ \\
\hline Xylopia humblotiana Baill. & Hazoambo & $L$ & $\begin{array}{l}\text { Back pain, stomach pain, } \\
\text { fatigue, urine retention, dizziness }\end{array}$ & $E$ \\
\hline \multicolumn{5}{|l|}{ APHLOIACEAE } \\
\hline \multirow[t]{2}{*}{ Aphloia theiformis (Vahl) Benn. } & \multirow[t]{2}{*}{ Fandramanana } & S & $\begin{array}{l}\text { Dental cavity, fatigue, muscle } \\
\text { pain }\end{array}$ & \multirow[t]{2}{*}{ C, M, S, Af } \\
\hline & & $\mathrm{L}$ & Wounds, burns, boils & \\
\hline \multicolumn{5}{|l|}{ APIACEAE } \\
\hline Centella asiatica (L.) Urb. & Viliantsahona & $\mathrm{L}$ & Boils & $\mathrm{Na}$ \\
\hline \multicolumn{5}{|l|}{ APOCYNACEAE } \\
\hline Catharanthus roseus (L.) G.Don & $\begin{array}{l}\text { Vonenina, } \\
\text { revitambelona }\end{array}$ & $E$ & $\begin{array}{l}\text { Cancer, stomach pain, urine } \\
\text { retention, pancreas problems }\end{array}$ & $E$ \\
\hline \multirow[t]{4}{*}{ Tabernaemontana ciliata Pichon } & \multirow[t]{4}{*}{ Intona } & $\mathrm{B}$ & Stomach pain, malaria & \multirow[t]{4}{*}{$E$} \\
\hline & & $L$ & Stomach pain & \\
\hline & & $L, S$ & Abdominal pain & \\
\hline & & $S$ & Intestinal parasites & \\
\hline \multicolumn{5}{|l|}{ AQUIFOLIACEAE } \\
\hline Ilex mitis (L.) Radlk. & Hazondrano & $L$ & Back pain & Af \\
\hline
\end{tabular}




\begin{tabular}{|c|c|c|c|c|}
\hline Scientific Names & Vernacular Names & $\begin{array}{l}\text { Part } \\
\text { used }\end{array}$ & Disease/Use & Distribution \\
\hline \multicolumn{5}{|l|}{ ARACEAE } \\
\hline $\begin{array}{l}\text { Typhonodorum lindleyanum } \\
\text { Schott }\end{array}$ & Via & $S$ & Wounds & C, M, Af \\
\hline \multicolumn{5}{|l|}{ ARECACEAE } \\
\hline Raphia sp. & Rofia & L & Wounds & \\
\hline \multicolumn{5}{|l|}{ ASPARAGACEAE } \\
\hline Dracaena reflexa Lam. & Hasina & RF & Boils & $M, A f$ \\
\hline \multicolumn{5}{|l|}{ ASTERACEAE } \\
\hline Acanthospermum hispidum DC. & Bakakely & L & $\begin{array}{l}\text { Diarrhea, abdominal pain, } \\
\text { wounds }\end{array}$ & Af \\
\hline Elephantopus mollis Kunth & Angadoha & L & Intestinal parasites & $\mathrm{Cu}$ \\
\hline Helianthus annuus $\mathrm{L}$. & Tanamasoandro & $\mathrm{L}$ & Intestinal parasites & $\mathrm{Cu}$ \\
\hline \multirow{2}{*}{$\begin{array}{l}\text { Humbertacalia leucopappa (DC.) } \\
\text { C.Jeffrey }\end{array}$} & \multirow[t]{2}{*}{ Vorovoka } & $\mathrm{L}$ & Gonorrhea & \multirow[t]{2}{*}{$E$} \\
\hline & & $L, S$ & Urine retention & \\
\hline $\begin{array}{l}\text { Oliganthes pseudocentauropsis } \\
\text { (Humbert) Humbert }\end{array}$ & Hazomboay & B & Malaria, dental cavity & $E$ \\
\hline \multirow[t]{3}{*}{ Psiadia altissima (DC.) Drake } & \multirow[t]{3}{*}{ Dingadingana } & $\mathrm{F}$ & Deafness & \multirow[t]{3}{*}{$E$} \\
\hline & & $\mathrm{B}$ & Dental cavity & \\
\hline & & $S$ & Intestinal parasites & \\
\hline Vernonia appendiculata Less. & Antsotry & L & Dental cavity & $\mathrm{E}$ \\
\hline \multicolumn{5}{|l|}{ BIGNONIACEAE } \\
\hline Phyllarthron bojeranum DC. & Zahana & L & $\begin{array}{l}\text { Urine retention, stomach pain, } \\
\text { fatigue, sexual problems, back } \\
\text { pain, dizziness }\end{array}$ & $E$ \\
\hline \multicolumn{5}{|l|}{ BRASSICACEAE } \\
\hline Nasturtium officinale W.T.Aiton & Anandrano & L & Hypertension & $\mathrm{Cu}$ \\
\hline \multicolumn{5}{|l|}{ BROMELIACEAE } \\
\hline Ananas comosus (L.) Merr. & Mananasy & Bud & Urine retention & $\mathrm{Cu}$ \\
\hline \multicolumn{5}{|l|}{ CANELLACEAE } \\
\hline \multirow[t]{3}{*}{ Cinnamosma fragrans Baill. } & \multirow[t]{2}{*}{ Fanalamangidy } & L, F & Intestinal parasites & \multirow[t]{3}{*}{$E$} \\
\hline & & L & Dental cavity & \\
\hline & Mandravasarotra & $S$ & Abdominal pain & \\
\hline \begin{tabular}{|l} 
Cinnamosma madagascariensis \\
Danguy
\end{tabular} & Sakarivohazo & $\mathrm{R}$ & Diarrhea & $E$ \\
\hline \multicolumn{5}{|l|}{ CANNABACEAE } \\
\hline \multirow[t]{2}{*}{ Trema orientalis (L.) Blume } & \multirow[t]{2}{*}{ Vakoka } & Seed & Dental cavity & \multirow[t]{2}{*}{ Af } \\
\hline & & $\mathrm{L}$ & Boil & \\
\hline \multicolumn{5}{|l|}{ CARICACEAE } \\
\hline \multirow[t]{3}{*}{ Carica papaya L. } & \multirow[t]{3}{*}{ Mampaza } & L & $\begin{array}{l}\text { Stomach pain, dental cavity, } \\
\text { urine retention, breast tumor }\end{array}$ & \multirow[t]{3}{*}{$\mathrm{Cu}$} \\
\hline & & $\mathrm{R}$ & Gonorrhea & \\
\hline & & $\mathrm{F}$ & Bilharzia & \\
\hline
\end{tabular}



men in Ambalabe, Madagascar

\begin{tabular}{|c|c|c|c|c|}
\hline Scientific Names & Vernacular Names & $\begin{array}{l}\text { Part } \\
\text { used }\end{array}$ & Disease/Use & Distribution \\
\hline \multicolumn{5}{|l|}{ CHRYSOBALANACEAE } \\
\hline Grangeria porosa Boivin ex Baill. & Maitsoririnina & $\mathrm{L}$ & Urine retention & $\mathrm{E}$ \\
\hline \multicolumn{5}{|l|}{ COMBRETACEAE } \\
\hline Terminalia sp. & Atafanala & $\mathrm{L}$ & Back pain & \\
\hline \multicolumn{5}{|l|}{ COMMELINACEAE } \\
\hline $\begin{array}{l}\text { Rhopalephora rugosa (H.Perrier) } \\
\text { Faden }\end{array}$ & Lomanorano & $\mathrm{F}$ & Cough & E \\
\hline \multicolumn{5}{|l|}{ CONNARACEAE } \\
\hline \multirow[t]{3}{*}{ Agelaea pentagyna (Lam.) Baill. } & \multirow[t]{3}{*}{ Vahimintina } & $S$ & $\begin{array}{l}\text { Malaria, abdominal pain, sexual } \\
\text { problems, vision problems }\end{array}$ & \multirow[t]{3}{*}{ C, M, Af } \\
\hline & & $B$ & Wound & \\
\hline & & L, S & Gonorrhea & \\
\hline \multicolumn{5}{|l|}{ CRASSULACEAE } \\
\hline $\begin{array}{l}\text { Kalanchoe prolifera (Bowie } \\
\text { ex Hook.) Raym.-Hamet }\end{array}$ & Sodifafana & L & Cough & E \\
\hline \multicolumn{5}{|l|}{ CUCURBITACEAE } \\
\hline Cucurbita maxima Duchesne & Pongy & $\mathrm{L}$ & Urine retention & $\mathrm{Na}$ \\
\hline \multicolumn{5}{|l|}{ CYATHEACEAE } \\
\hline Cyathea marattioides Willd. & Fanjana, Faho & $\mathrm{L}$ & Back pain & $\mathrm{E}$ \\
\hline \multicolumn{5}{|l|}{ DILLENIACEAE } \\
\hline Dillenia triquetra (Rottb.) Gilg & Bararaka & $\mathrm{L}$ & Sexual problems & E \\
\hline $\begin{array}{l}\text { Tetracera madagascariensis } \\
\text { Willd. ex Schltdl. }\end{array}$ & Vahimarana & $L$ & Intestinal parasites, back pain & $E$ \\
\hline \multicolumn{5}{|l|}{ ERYTHROXYLACEAE } \\
\hline \multirow[t]{3}{*}{ Erythroxylum sp. } & \multirow[t]{3}{*}{ Menahihy } & $\mathrm{L}$ & Stomach pain & \\
\hline & & $B$ & Diarrhea & \\
\hline & & $S$ & Stomach pain & \\
\hline \multicolumn{5}{|l|}{ EUPHORBIACEAE } \\
\hline \multirow[t]{2}{*}{ Euphorbia hirta L. } & \multirow[t]{2}{*}{ Jean Robert } & $S$ & Gonorrhea & \multirow[t]{2}{*}{$\mathrm{Na}$} \\
\hline & & $\mathrm{L}$ & Urine retention & \\
\hline Euphorbia sp. & Tanatanamanga & L & $\begin{array}{l}\text { Pancreas problems, increase } \\
\text { blood protein content }\end{array}$ & \\
\hline \multirow[t]{2}{*}{ Hura crepitans L. } & \multirow[t]{2}{*}{ Hazomboay } & $\mathrm{L}$ & Dental cavity, wound & \multirow[t]{2}{*}{ NE } \\
\hline & & $\mathrm{B}$ & Diarrhea & \\
\hline \multirow[t]{2}{*}{ Macaranga alnifolia Baker } & \multirow[t]{2}{*}{ Mankaranana } & $B$ & Diarrhea & \multirow[t]{2}{*}{$E$} \\
\hline & & Latex & Boil & \\
\hline Manihot esculenta Crantz & Mangahazo & L & Intestinal parasites & $\mathrm{Cu}$ \\
\hline \multicolumn{5}{|l|}{ FABACEAE } \\
\hline Abrus precatorius $\mathrm{L}$. & Voamaintilany & $\mathrm{B}$ & Cough & Af, As \\
\hline Cajanus sp. & Antsotry morona & L, S & Urine retention & \\
\hline Cajanus sp. & Antsotry & L & Boils & \\
\hline
\end{tabular}




\begin{tabular}{|c|c|c|c|c|}
\hline Scientific Names & Vernacular Names & $\begin{array}{l}\text { Part } \\
\text { used }\end{array}$ & Disease/Use & Distribution \\
\hline \multirow{2}{*}{$\begin{array}{l}\text { Chamaecrista mimosoides (L.) } \\
\text { Greene }\end{array}$} & \multirow[t]{2}{*}{ Ramirina } & $\mathrm{L}$ & Diarrhea, bilharzia & \multirow[t]{2}{*}{$\mathrm{NE}$} \\
\hline & & $s$ & Fatigue & \\
\hline Clitoria lasciva Bojer ex Benth. & Vahitsikomba & $S$ & Wound & $\mathrm{E}$ \\
\hline \multirow{2}{*}{$\begin{array}{l}\text { Crotalaria xanthoclada Bojer ex } \\
\text { Benth. }\end{array}$} & \multirow[t]{2}{*}{ Maitsoririnina } & L, S & Diarrhea, intestinal parasites & \multirow[t]{2}{*}{ Af } \\
\hline & & $L$ & Wound & \\
\hline $\begin{array}{l}\text { Desmodium ramosissimum } \\
\text { G.Don }\end{array}$ & Tsilavondrivotra & $L$ & $\begin{array}{l}\text { Diarrhea, abdominal pain, dental } \\
\text { cavity }\end{array}$ & NE \\
\hline Entada gigas (L.) Fawc. \& Rendle & Vahinkarabo & $s$ & Boils, diarrhea & $\mathrm{NE}$ \\
\hline Millettia hitsika Du Puy \& Labat & Hitsika & & Bilharzia & $E$ \\
\hline Phylloxylon sp. & Harahara, arahara & $\mathrm{L}$ & $\begin{array}{l}\text { Stomach pain, fatigue, urine } \\
\text { retention }\end{array}$ & $E$ \\
\hline \multicolumn{5}{|l|}{ GENTIANACEAE } \\
\hline \multirow[t]{2}{*}{ Exacum quinquenervium Griseb. } & Mamoahely & $\mathrm{R}$ & Malaria & \multirow[t]{2}{*}{ M } \\
\hline & & $B$ & Back pain & \\
\hline \multicolumn{5}{|l|}{ HYPERICACEAE } \\
\hline \multirow{3}{*}{$\begin{array}{l}\text { Harungana madagascariensis } \\
\text { Lam. ex Poir. }\end{array}$} & \multirow[t]{3}{*}{ Harongana } & $B$ & Diarrhea & \multirow[t]{3}{*}{ C, M, Af } \\
\hline & & $L$ & $\begin{array}{l}\text { Intestinal parasites, urine } \\
\text { retention, increase blood protein } \\
\text { content }\end{array}$ & \\
\hline & & $S$ & Vision problems & \\
\hline \multicolumn{5}{|l|}{ LAMIACEAE } \\
\hline Cassytha filiformis $\mathrm{L}$. & Maroampototra & s & Dental cavity & 1 \\
\hline $\begin{array}{l}\text { Clerodendrum aucubifolium } \\
\text { Baker }\end{array}$ & Atambalahy & L & Wound & $E$ \\
\hline Clerodendrum sp. & Sifontsohy & $L$ & Urine retention & \\
\hline Ocimum gratissimum L. & Romba & L & Dental cavity, cough & $\begin{array}{l}\text { C, M, S, } \\
\text { Af, As }\end{array}$ \\
\hline \multicolumn{5}{|l|}{ LYCOPODIACEAE } \\
\hline \multirow[t]{3}{*}{ Lycopodium clavatum L. } & \multirow[t]{3}{*}{ Tanatrandraka } & L, S & Urine retention & \multirow[t]{3}{*}{1} \\
\hline & & L & Boil, stomach pain & \\
\hline & & $R, L$ & Malaria & \\
\hline \multicolumn{5}{|l|}{ LYGODIACEAE } \\
\hline Lygodium lanceolatum Desv. & Famatotrakanga & L & $\begin{array}{l}\text { Diarrhea, pancreas } \\
\text { problems, hypertension, } \\
\text { fatigue, hemorrhoids }\end{array}$ & 1 \\
\hline \multicolumn{5}{|l|}{ MALVACEAE } \\
\hline Pavonia urens Cav. & Tsontsona lahy & $\mathrm{L}$ & Boils & Af \\
\hline \multicolumn{5}{|l|}{ MELASTOMATACEAE } \\
\hline \multirow[t]{2}{*}{ Clidemia hirta (L.) D.Don } & \multirow[t]{2}{*}{ Mazambody } & $\mathrm{R}$ & Stomach pains & \multirow[t]{2}{*}{$\mathrm{Na}$} \\
\hline & & $\mathrm{L}$ & Wounds & \\
\hline Tristemma mauritianum J.F.Gmel. & Voatrotroka & $L$ & $\begin{array}{l}\text { Abdominal pain, bilharzia, } \\
\text { urine retention }\end{array}$ & C, Af \\
\hline
\end{tabular}



men in Ambalabe, Madagascar

\begin{tabular}{|c|c|c|c|c|}
\hline Scientific Names & Vernacular Names & $\begin{array}{l}\text { Part } \\
\text { used }\end{array}$ & Disease/Use & Distribution \\
\hline \multicolumn{5}{|l|}{ MELIACEAE } \\
\hline \multirow[t]{3}{*}{ Melia azedarach $\mathrm{L}$. } & \multirow[t]{3}{*}{ Voandelaka } & $L, R$ & Pancreas problems & \multirow[t]{3}{*}{$\mathrm{Na}$} \\
\hline & & B & Intestinal parasites & \\
\hline & & $\mathrm{L}$ & Fatigue & \\
\hline \multicolumn{5}{|l|}{ MENISPERMACEAE } \\
\hline Strychnopsis thouarsii Baill. & Tanteliravina & $\mathrm{R}$ & Gonorrhea & $E$ \\
\hline \multicolumn{5}{|l|}{ MOLLUGINACEAE } \\
\hline \multirow[t]{3}{*}{ Mollugo nudicaulis Lam. } & \multirow[t]{3}{*}{ Aferontany } & $\mathrm{L}$ & $\begin{array}{l}\text { Increase blood protein content, } \\
\text { cough }\end{array}$ & \multirow[t]{3}{*}{$\mathrm{Au}, \mathrm{Af}, \mathrm{N}$} \\
\hline & & $E$ & $\begin{array}{l}\text { Malaria, diarrhea, abdominal } \\
\text { pain, infection during pregnancy }\end{array}$ & \\
\hline & & B & Burns & \\
\hline \multicolumn{5}{|l|}{ MONIMIACEAE } \\
\hline Tambourissa thouvenotii Danguy & Ambora maventy & $\mathrm{L}$ & Urine retention & $E$ \\
\hline \multicolumn{5}{|l|}{ MORACEAE } \\
\hline Artocarpus integer (Thunb.) Merr. & Ampalibe & $\mathrm{L}$ & Diarrhea & NE \\
\hline Artocarpus sp. & Frampe & & Asthma & \\
\hline Ficus sycomorus L. & Voara & $\mathrm{F}$ & Intestinal parasites & C, Af \\
\hline \multirow[t]{2}{*}{ Ficus polita Vahl } & \multirow[t]{2}{*}{ Mandrisy } & $\mathrm{L}$ & Stomach pain, urine retention & \multirow[t]{2}{*}{ Af } \\
\hline & & $E$ & Fatigue & \\
\hline Ficus polyphlebia Baker & Voararano & $\mathrm{L}$ & Wound, gonorrhea & $E$ \\
\hline Ficus pyrifolia Burm.f. & Nonoka & $\mathrm{L}$ & Cough, wounds & $E$ \\
\hline Ficus politoria Lam. & Ramiringitra & $\mathrm{B}$ & Boils & $E$ \\
\hline Morus alba L. & Voandroy & $\mathrm{L}$ & Stomach pain, urine retention & $\mathrm{Na}$ \\
\hline \multicolumn{5}{|l|}{ MUSACEAE } \\
\hline \multirow[t]{2}{*}{ Musa sp. } & \multirow[t]{2}{*}{ Akondro } & $\mathrm{L}$ & Diarrhea & \\
\hline & & Trunk & Abdominal pain & \\
\hline \multicolumn{5}{|l|}{ MORACEAE } \\
\hline Streblus sp. & Dipaty & $\mathrm{L}$ & Wounds, urine retention & \\
\hline \multicolumn{5}{|l|}{ MYRISTICACEAE } \\
\hline \multirow{2}{*}{$\begin{array}{l}\text { Mauloutchia humblotii (H.Perrier) } \\
\text { Capuron }\end{array}$} & \multirow[t]{2}{*}{ Ilondraharaha } & $\mathrm{L}$ & Cough & \multirow[t]{2}{*}{$E$} \\
\hline & & $\mathrm{F}$ & Wound & \\
\hline \multicolumn{5}{|l|}{ MYRTACEAE } \\
\hline Eucalyptus sp. & Kininina & Bud & Abdominal pain & \\
\hline $\begin{array}{l}\text { Myrcianthes fragrans (Sw.) } \\
\text { McVaugh }\end{array}$ & Jirofo & $L$ & Malaria, dental cavity & $N$ \\
\hline Eugenia goviala H.Perrier & Gavoala & $L$ & $\begin{array}{l}\text { Stomach pain, diarrhea, dental } \\
\text { cavity }\end{array}$ & $E$ \\
\hline Eugenia sp. & Rotra & B & Diarrhea & \\
\hline Psidium cattleyanum Sabine & $\begin{array}{l}\text { Gavo madinika, } \\
\text { goavy }\end{array}$ & B & $\begin{array}{l}\text { Diarrhea, abdominal pain, } \\
\text { dental cavity }\end{array}$ & $\mathrm{Na}$ \\
\hline $\begin{array}{l}\text { Syzygium bernieri (Drake) Labat } \\
\text { \& G.E.Schatz }\end{array}$ & Goaviala & Bud & Stomach pain & $E$ \\
\hline
\end{tabular}




\begin{tabular}{|c|c|c|c|c|}
\hline Scientific Names & Vernacular Names & $\begin{array}{l}\text { Part } \\
\text { used }\end{array}$ & Disease/Use & Distribution \\
\hline \multicolumn{5}{|l|}{ OLEACEAE } \\
\hline Noronhia gracilipes H.Perrier & Tsilaitra & $\mathrm{L}$ & Malaria, dizziness & $E$ \\
\hline \multicolumn{5}{|l|}{ ONAGRACEAE } \\
\hline $\begin{array}{l}\text { Ludwigia octovalvis (Jacq.) } \\
\text { P.H.Raven }\end{array}$ & Tongobintsy & L & Intestinal parasites & Af, $N$ \\
\hline \multicolumn{5}{|l|}{ PANDANACEAE } \\
\hline Pandanus sp. & Vakoana & $\mathrm{L}$ & Fatigue & \\
\hline \multicolumn{5}{|l|}{ PHYLLANTHACEAE } \\
\hline $\begin{array}{l}\text { Phyllanthus amarus Schumach. } \\
\text { \& Thonn. }\end{array}$ & Mandrihariva & $E$ & Stomach pain & $\mathrm{Na}$ \\
\hline Uapaca bojeri Baill. & Vakoana & $R$ & Sexual problems & $E$ \\
\hline Uapaca sp. & Voampaka kely & $L$ & Fatigue & \\
\hline \multicolumn{5}{|l|}{ PHYSENACEAE } \\
\hline Physena sp. & Fanamamangidy & $S$ & Toxicity & $\mathrm{E}$ \\
\hline \multicolumn{5}{|l|}{ PIPERACEAE } \\
\hline Piper sp. & Tsimalatsaka & $B$ & Gonorrhea & \\
\hline \multicolumn{5}{|l|}{ PITTOSPORACEAE } \\
\hline \begin{tabular}{|l} 
Pittosporum sp. \\
\end{tabular} & Sanganakoholahy & $\mathrm{L}$ & Urine retention & \\
\hline \multicolumn{5}{|l|}{ POACEAE } \\
\hline \multirow[t]{2}{*}{ Imperata cylindrica (L.) Raeusch. } & \multirow[t]{2}{*}{ Tenona } & $L$ & $\begin{array}{l}\text { Fatigue, hypertension, boils, } \\
\text { abdominal pain, hemorrhoids }\end{array}$ & \multirow[t]{2}{*}{$\mathrm{Na}$} \\
\hline & & $E$ & Urine retention & \\
\hline Panicum sp. & Ahipody & & Dental cavity & \\
\hline Sporobolus pyramidalis P.Beauv. & Ahidroranga & $\mathrm{L}$ & Back pain & $\mathrm{Na}$ \\
\hline Zea mays $\mathrm{L}$. & Katsaka & $\mathrm{F}$ & Urine retention & $\mathrm{Cu}$ \\
\hline \multicolumn{5}{|l|}{ POLYGONACEAE } \\
\hline \multirow{3}{*}{$\begin{array}{l}\text { Persicaria senegalensis (Meisn.) } \\
\text { Soják }\end{array}$} & \multirow[t]{3}{*}{ Arivotoambelona } & $\mathrm{R}$ & Malaria, abdominal pain & \multirow[t]{3}{*}{$\mathrm{Na}$} \\
\hline & & $\mathrm{R}, \mathrm{L}$ & Diarrhea & \\
\hline & & $\mathrm{L}$ & Cancer & \\
\hline \multicolumn{5}{|l|}{ PRIMULACEAE } \\
\hline Embelia concinna Baker & Takasina & B & Cough & $E$ \\
\hline $\begin{array}{l}\text { Oncotemum boivinianum } \\
\text { H.Perrier }\end{array}$ & Ramitsiaka & L & Wound & $E$ \\
\hline $\begin{array}{l}\text { Oncostemum palmiforme } \\
\text { H. Perrier }\end{array}$ & Kelimalaza & $S$ & Wound & $E$ \\
\hline \multicolumn{5}{|l|}{ ROSACEAE } \\
\hline \multirow{2}{*}{$\begin{array}{l}\text { Eriobotrya japonica (Thunb.) } \\
\text { Lindl. }\end{array}$} & \multirow[t]{2}{*}{ Pibasy } & $\mathrm{L}$ & Infection during pregnancy & \multirow[t]{2}{*}{$\mathrm{Cu}$} \\
\hline & & $S$ & Bilharzia & \\
\hline Rubus buergeri Miq. & Takoaka & $\mathrm{L}$ & Diarrhea, abdominal pain & $\mathrm{Cu}$ \\
\hline \multicolumn{5}{|l|}{ RUBIACEAE } \\
\hline \multirow[t]{2}{*}{$\begin{array}{l}\text { Chapelieria madagascariensis } \\
\text { A.Rich. ex DC. }\end{array}$} & \multirow[t]{2}{*}{ Ravimbolo } & $L$ & $\begin{array}{l}\text { Stomach pain, abdominal pain, } \\
\text { dental cavity, urine retention }\end{array}$ & \multirow[t]{2}{*}{$E$} \\
\hline & & $S, L$ & Back pain & \\
\hline
\end{tabular}



men in Ambalabe, Madagascar

\begin{tabular}{|c|c|c|c|c|}
\hline Scientific Names & Vernacular Names & $\begin{array}{l}\text { Part } \\
\text { used }\end{array}$ & Disease/Use & Distribution \\
\hline Chassalia sp. & Ahitrimpa & $\mathrm{L}$ & Stomach pain & \\
\hline Danais rhamnifolia Baker & Hazombary & $\mathrm{L}$ & Cough & $E$ \\
\hline $\begin{array}{l}\text { Diodella sarmentosa (Sw.) } \\
\text { Bacigalupo \& Cabral ex Borhidi }\end{array}$ & Lelamenarana & Latex & Wound & $\mathrm{Na}$ \\
\hline Gaertnera macrostipula Baker & Tsikafekafe & $L$ & Urine retention & $E$ \\
\hline \multirow[t]{3}{*}{ Paederia sp. } & Vahivola & $\mathrm{L}$ & Abdominal pain, cancer & \\
\hline & Vahilaingo & Latex & Dental cavity & \\
\hline & - & $\mathrm{B}$ & Gonorrhea & \\
\hline $\begin{array}{l}\text { Pauridiantha paucinervis (Hiern) } \\
\text { Bremek. }\end{array}$ & Tsiandrova & $L$ & Stomach pain, malaria & 1 \\
\hline $\begin{array}{l}\text { Peponidium humbertianum } \\
\text { (Cavaco) Razafim., Lantz \& } \\
\text { B.Bremer }\end{array}$ & Pitsikahitra & $L$ & Malaria, stomach pain & $E$ \\
\hline Psychotria sp. & Marovelo & $\mathrm{L}$ & Fatigue & \\
\hline \multicolumn{5}{|l|}{ RUTACEAE } \\
\hline \multirow[t]{2}{*}{ Citrus $\times$ aurantium $\mathrm{L}$. } & Vohangintsinoa & $\mathrm{F}$ & Cough & $\mathrm{Na}$ \\
\hline & Voahangiala & $\mathrm{L}$ & Cough & \\
\hline Toddalia asiatica (L.) Lam. & Anakasimba & $L$ & Stomach pain, back pain & $\begin{array}{l}\text { C, M, S, } \\
\text { Af, As }\end{array}$ \\
\hline \multirow[t]{2}{*}{ Vepris ampody H.Perrier } & \multirow[t]{2}{*}{ Ampody } & $\mathrm{L}$ & Abdominal pain & $\mathrm{E}$ \\
\hline & & $S$ & Wound & \\
\hline \multicolumn{5}{|l|}{ SALICACEAE } \\
\hline \multirow[t]{2}{*}{ Homalium axillare (Lam.) Benth. } & \multirow[t]{2}{*}{ Hazombato } & $B$ & Burns & \multirow[t]{2}{*}{$E$} \\
\hline & & $S$ & Back pain & \\
\hline \multirow[t]{2}{*}{ Homalium sp. } & \multirow[t]{2}{*}{ Tendrompony } & $\mathrm{R}$ & Back pain & \\
\hline & & $\mathrm{L}$ & Urine retention & \\
\hline \multicolumn{5}{|l|}{ SAPINDACEAE } \\
\hline $\begin{array}{l}\text { Allophylus decaryi Danguy \& } \\
\text { Choux }\end{array}$ & Mampe & $L$ & Asthma & $\mathrm{E}$ \\
\hline Allophylus sp. & Teloravina & $L$ & Vision problems & \\
\hline Litchi sinensis Sonn. & Letchi & $\mathrm{L}$ & Abdominal pain & $\mathrm{Cu}$ \\
\hline \multicolumn{5}{|l|}{ SAPOTACEAE } \\
\hline $\begin{array}{l}\text { Chrysophyllum boivinianum } \\
\text { (Pierre) Baehni }\end{array}$ & Famelona & $L$ & $\begin{array}{l}\text { Malaria, fatigue, increase blood } \\
\text { protein content, levels, muscle } \\
\text { pain }\end{array}$ & $\mathrm{C}$, \\
\hline \multicolumn{5}{|l|}{ SOLANACEAE } \\
\hline Nicotiana tabacum L. & Paraky gasy & $\mathrm{L}$ & Bilharzia & $\mathrm{Cu}$ \\
\hline Solanum mauritianum Scop. & Bakobako & $B$ & Abdominal pain & $\mathrm{Na}$ \\
\hline \multicolumn{5}{|l|}{ SPHAEROSEPALACEAE } \\
\hline \multirow{2}{*}{$\begin{array}{l}\text { Rhopalocarpus louvelii (Danguy) } \\
\text { Capuron }\end{array}$} & \multirow[t]{2}{*}{ Hazomamy } & $\mathrm{B}$ & Intestinal parasites & \multirow[t]{2}{*}{$\mathrm{E}$} \\
\hline & & $L$ & Toxicity & \\
\hline
\end{tabular}




\begin{tabular}{|c|c|c|c|c|}
\hline Scientific Names & Vernacular Names & $\begin{array}{l}\text { Part } \\
\text { used }\end{array}$ & Disease/Use & Distribution \\
\hline \multirow{2}{*}{$\begin{array}{l}\text { Nuxia oppositifolia (Hochst.) } \\
\text { Benth. }\end{array}$} & \multirow[t]{2}{*}{ Valanirana } & $\mathrm{L}$ & Stomach pain & \multirow[t]{2}{*}{ Af, As } \\
\hline & & $L$ & $\begin{array}{l}\text { Urine retention, pancreas } \\
\text { problems }\end{array}$ & \\
\hline \multicolumn{5}{|l|}{ STRELITZIACEAE } \\
\hline \multirow{2}{*}{$\begin{array}{l}\text { Ravenala madagascariensis } \\
\text { Sonn. }\end{array}$} & \multirow[t]{2}{*}{ Ravinala } & Heart & Stomach pain & \multirow[t]{2}{*}{$\mathrm{E}$} \\
\hline & & L & Cough, urine retention & \\
\hline \multicolumn{5}{|l|}{ THEACEAE } \\
\hline Camellia sinensis (L.) Kuntze & Tsiandrova & $\mathrm{L}$ & Fatigue, dizziness & $\mathrm{Na}$ \\
\hline \multicolumn{5}{|l|}{ VERBENACEAE } \\
\hline \multirow[t]{2}{*}{ Lantana camara L. } & \multirow[t]{2}{*}{ Radriaka } & L & $\begin{array}{l}\text { Malaria, wounds, sexual } \\
\text { problems, hypertension }\end{array}$ & \multirow[t]{2}{*}{$\mathrm{Na}$} \\
\hline & & $\mathrm{R}$ & Intestinal parasites & \\
\hline \multicolumn{5}{|l|}{ VITACEAE } \\
\hline Vitis vinifera $\mathrm{L}$. & Voalomboka & $\mathrm{F}$ & Urine retention & $\mathrm{Cu}$ \\
\hline \multicolumn{5}{|l|}{ XANTHORRHOEACEAE } \\
\hline Dianella ensifolia (L.) DC. & Herana & $\mathrm{R}$ & Abdominal pain & $\begin{array}{l}\text { M, S, Au, } \\
\text { Af, As }\end{array}$ \\
\hline Aloe macroclada Baker & Vahona & L & Stomach pain & $\mathrm{E}$ \\
\hline \multicolumn{5}{|l|}{ ZINGIBERACEAE } \\
\hline $\begin{array}{l}\text { Aframomum angustifolium } \\
\text { (Sonn.) K.Schum. }\end{array}$ & Longoza & L & Intestinal parasites & Af \\
\hline Curcuma longa L. & Tamotamo & $\mathrm{R}$ & Malaria, yellow fever & $\mathrm{Cu}$ \\
\hline
\end{tabular}

medicinal plants were reported: barking, gathering leaves, cutting branches, and uprooting the whole plant.

Nearly 30 diseases were listed as having medicinal plant treatments. Commonly mentioned diseases were malaria, sexual problems, urinary retention, muscle aches, fatigue, dental problems, diarrhea, cough, and bilharzia
(Table 2). It was very common for one species to be used to treat two or more diseases. For example: dental cavity, wounds, burns, boils, fatigue, and muscle pain are all treated with Aphloia theiformis (Vahl) Benn. Similarly two or more species may be utilized in the treatment of a single disease. For example, malaria is treated with Exacum

Table 2. Diseases known to have a medicinal plant treatment by men from Amabale, Madagascar

\begin{tabular}{|l|c|}
\hline Disease & $\begin{array}{c}\text { \# of species } \\
\text { as treatment }\end{array}$ \\
\hline Abdominal pain & 20 \\
\hline Asthma & 2 \\
\hline Back pain & 13 \\
\hline Bilharzia & 7 \\
\hline Boils & 9 \\
\hline Burns & 3 \\
\hline Cancer & 4 \\
\hline Cough & 12 \\
\hline Deafness & 1 \\
\hline Dental cavity & 18 \\
\hline
\end{tabular}

\begin{tabular}{|l|c|}
\hline Disease & $\begin{array}{c}\text { \# of species } \\
\text { as treatment }\end{array}$ \\
\hline Diarrhea & 20 \\
\hline Dizziness & 4 \\
\hline Fatigue & 14 \\
\hline Gonorrhea & 9 \\
\hline Hemorrhoids & 3 \\
\hline Hypertension & 4 \\
\hline $\begin{array}{l}\text { Increase blood } \\
\text { protein content }\end{array}$ & 4 \\
\hline $\begin{array}{l}\text { Infection during } \\
\text { pregnancy }\end{array}$ & 2 \\
\hline Intestinal parasites & 16 \\
\hline
\end{tabular}

\begin{tabular}{|l|c|}
\hline Disease & $\begin{array}{c}\text { \# of species } \\
\text { as treatment }\end{array}$ \\
\hline Malaria & 14 \\
\hline Muscle pain & 2 \\
\hline Pancreas pain & 5 \\
\hline Sexual problems & 6 \\
\hline Stomach pain & 25 \\
\hline Toxicity & 2 \\
\hline Urine retention & 30 \\
\hline Vision problems & 3 \\
\hline Wounds & 7 \\
\hline Yellow fever & 1 \\
\hline
\end{tabular}




\section{Rabearivony et al. - Ethnobotanical study of the medicinal plants known by men in Ambalabe, Madagascar}

Table 3. Species from Vohibe Forest, Madagascar, with highest Use Index (I\%) with additional uses and ecological status. IUCN Status (IUCN Red List 2013): endangered (E), vulnerable (V).

\begin{tabular}{|c|c|c|c|c|}
\hline Species & Distribution & $\begin{array}{l}\text { IUCN } \\
\text { status }\end{array}$ & $1 \%$ & Local use \\
\hline $\begin{array}{l}\text { Dillenia triquetra (Rottb.) } \\
\text { Gilg }\end{array}$ & Endemic & $E$ & 94 & $\begin{array}{l}\text { Used in the construction of houses. It is in high demand } \\
\text { by men with sexual problems and is seen as an } \\
\text { effective stimulant. }\end{array}$ \\
\hline $\begin{array}{l}\text { Noronhia gracilipes } \\
\text { H.Perrier }\end{array}$ & Endemic & $E$ & 92 & $\begin{array}{l}\text { This species is highly sought after by locals because of } \\
\text { the hardness of its wood. Used in the treatment of } \\
\text { diseases such as malaria. }\end{array}$ \\
\hline $\begin{array}{l}\text { Chrysophyllum boivinianum } \\
\text { (Pierre) Baehni }\end{array}$ & Comoros & V & 88 & $\begin{array}{l}\text { This species is highly prized by locals for the treatment } \\
\text { of many diseases. Locally used to construct houses. }\end{array}$ \\
\hline Xylopia humblotiana Baill. & Endemic & $E$ & 86 & $\begin{array}{l}\text { This species is highly sought after as medicine. It is } \\
\text { also used in the construction of houses. }\end{array}$ \\
\hline Phyllarthron bojeranum DC. & Endemic & $E$ & 85 & $\begin{array}{l}\text { This species is widely used by men as a medicinal } \\
\text { tonic. It is highly valued by local people for making } \\
\text { canes for the tangalamena. }\end{array}$ \\
\hline $\begin{array}{l}\text { Mauloutchia humblotii } \\
\text { (H.Perrier) Capuron }\end{array}$ & Endemic & $E$ & 85 & $\begin{array}{l}\text { This species is highly valued for treating injuries during } \\
\text { circumcision. It is used to make canoes, and the oil is } \\
\text { used in the hair. }\end{array}$ \\
\hline $\begin{array}{l}\text { Aphloia theiformis } \\
\text { (Vahl) Benn. }\end{array}$ & $\begin{array}{l}\text { Comoros, } \\
\text { Mascarenes } \\
\text { Seychelles, } \\
\text { Africa }\end{array}$ & $\mathrm{V}$ & 82 & $\begin{array}{l}\text { Locally, this species is used for treating many diseases } \\
\text { including injury during circumcision. People use this } \\
\text { species for timber and firewood. }\end{array}$ \\
\hline Macaranga alnifolia Baker & Endemic & V & 81 & $\begin{array}{l}\text { Used as treatment of diarrhea and boils. It plays an } \\
\text { important role in the coming of age rituals and customs } \\
\text { as well as in the manufacture of canes. }\end{array}$ \\
\hline Eugenia goviala H.Perrier & Endemic & $E$ & 79 & $\begin{array}{l}\text { This species is used to treat certain diseases like tooth } \\
\text { decay, diarrhea, and stomach pain. It is highly prized for } \\
\text { the construction of furniture. }\end{array}$ \\
\hline $\begin{array}{l}\text { Oncostemum boivinianum } \\
\text { H.Perrier }\end{array}$ & Endemic & $E$ & 74 & $\begin{array}{l}\text { Used for treatment of injuries during circumcision. } \\
\text { Also used as firewood. }\end{array}$ \\
\hline $\begin{array}{l}\text { Peponidium humbertianum } \\
\text { (Cavaco) Razafim., Lantz \& } \\
\text { B.Bremer }\end{array}$ & Endemic & $E$ & 71 & $\begin{array}{l}\text { Used for the treatment of diseases like malaria and } \\
\text { stomach pain, as well as timber. }\end{array}$ \\
\hline $\begin{array}{l}\text { Fenerivia ghesquiereana } \\
\text { (Cavaco \& Keraudren) } \\
\text { R.M.K.Saunders }\end{array}$ & Endemic & $E$ & 69 & $\begin{array}{l}\text { Highly prized by men with sexual problems and } \\
\text { sexually transmitted diseases like gonorrhea. People } \\
\text { use this species for timber, wood manufacturing, and } \\
\text { wood plank cooking. }\end{array}$ \\
\hline
\end{tabular}

quinquenervium Griseb., Lycopodium clavatum L., and Myrcianthes fragrans (Sw.) McVaugh.

\section{Use Index}

Among the 137 species recorded, 12 species had Use Index (1\%) ratings at $60 \%$ or higher, showing they were most well-known within the community. The rate of endemism within the most well-known species is $83 \%$. The uses cited for these well-known species are not only medicinal but also for other realms of livelihood such as construction and rituals. Table 3 shows the IUCN status, as well as other uses and importance, of these species to the community.

\section{Discussion}

We have indication that our hypothesis of gendered use and knowledge of medicinal plants was supported as the men cited medicinal species used against the retention of urine, muscle pain, and fatigue and as aphrodisiacs, far more (and in the case of aphrodisiac, exclusively a male use category) than the women (Raveloson, forthcoming). However, the male pharmacopeia was not restricted to male diseases as mentions of plants to treat pregnancy 
complications as well as general diseases like malaria and cancer were also present.

Plant collecting methods reported by interviewees (gathering bark and leaves as well as cutting branches and uprooting the whole plant) give some cause for conservation concern. The removal of the bark in large quantities can destroy the plant because this part of the plant can no longer play the role of protector. Pulling up roots causes the total destruction of the plant (as is common in the collection of herbs and shrubs). These methods of gathering thus strongly undermine the sustainability of medicinal species use. The collection of leaves does not affect plant health as severely if the amount recovered is limited. Similarly, cutting the branches should not have negative impacts if the amount collected is reasonable. However, male Ambalabe collectors tend to strip the whole plant instead of focusing strictly on the necessary part.

While some studies have reported a low rate of use of endemic species as medicinal plants (Shangali et al. 2008), a similar study in Madagascar found a high rate of endemism and nativity in locally used medicinal species (Razafindraibe et al. 2013). A high rate of endemism in useful plants could be expected in a flora as unique as Madagascar with an $82 \%$ overall rate of endemism (Callmander et al. 2013), especially since many of the species with high calculated $1 \%$ are tree species, which have a higher rate of endemism in Madagascar (92\%) (Callmander et al. 2013).

While the use of endemic species is high it does not necessarily imply the species are rare. Vohibe Forest is a low to mid-elevation rainforest along the eastern escarpment of Madagascar, a dominant ecosystem in the region and part of a large corridor of humid rainforest. A rudimentary look at the IUCN Red List status within Madagascar (2013) of these widely known species shows that most of them are categorized as endangered or vulnerable. While risk is not extremely high, there is some concern for the conservation status and future status for these species if used unsustainably.

High levels of use of these largely endemic plant species could prove problematic if done unsustainably, especially those species with multiple use categories (medicine, ritual, timber, construction, etc.). A more in-depth study on how these endemic species are used and selected, as well as a comparison study of other sites within Madagascar, is needed to further understand the uniqueness of this traditional knowledge while a closer look at the local conservation status is needed to build a sustainable use plan.

\section{Conclusions}

The use of medicinal plants is still a common practice especially in rural areas of Madagascar. Moreover, the eco- nomic difficulties of the local people and the lack of basic health facilities promote the use of traditional medicine with medicinal plants. Based on the ethnobotanical surveys conducted among villagers, the majority of the population in Ambalabe employs the use of medicinal plants to treat diseases and maintain good health. The rate of endemism of the medicinal plants used by men from Vohibe Forest is 35\%. Our study found the most well-known medicinal species have an even higher rate of endemism $(83 \%)$. While many of these species are used to treat nonlife threatening illnesses, they are also used for firewood, construction materials, and for rituals. The intense harvesting of leaves in traditional medicine does not present an alarming threat to the forest species, although the rate of use is enormous (68\%). However, the utilization of bark $(10 \%)$ is quite problematic. Furthermore, the use of roots even at a low rate $(6 \%)$, especially the practice of completely uprooting a plant, constitutes a severe threat to the species.

The island nation of Madagascar has a high rate of endemic plant species. In Ambalabe, the use of plant species for daily living needs carries its own set of implications on the health of the natural resources. There is a relationship between the part of the plant used and the health of the plant population (Cunningham 1996). Information on pressures and threats on well-known and widely used species and their habitat are necessary for the conservation of these species, which in turn protects biodiversity and helps secure traditional practice in the future. Most threats to these species stem from human activities such as traditional slash and burn agriculture (tavy), overharvesting of plants with therapeutic properties, and overharvesting of timber (house building, canoe building, and craft work). The method of plant collecting and the heavy use of some species may contribute to the degradation of plant biodiversity and the health of Vohibe Forest and Ambalabe Commune. A deeper study on biological and ecological characteristics of species would be essential, especially for the most exploited species, in order to establish a better sense of potential biodiversity and traditional plant loss if conservation methods are not put into place.

\section{Acknowledgments}

We express our sincere thanks and gratitude to individuals and organizations who contributed to this study: Missouri Botanical Garden (MBG), which has collaborated closely with the Department of Plant Biology and Ecology, Faculty of Sciences of the University of Antananarivo; the WLBC for the financial funding of this study; the local villagers of Ambalabe; the team and managers at the MBG Ambalabe site; and all local guides.

\section{Literature Cited}




\section{Rabearivony et al. - Ethnobotanical study of the medicinal plants known by men in Ambalabe, Madagascar}

Andriamaholy, V.L. 1994. Etude de la Flore Médicinale de la Forêt Relicte d'Antananarivokely: Etude écologique, répartition, description des espèces et utilisations en pharmacopée traditionnelle. M.Sc. thesis, Departent of Biology, University of Antananarivo, Antananarivo, Madagascar.

Ayantunde, A.A., M. Briejer, P. Hiernaux, H.M.J. Udo \& R. Tabo. 2008. Botanical knowledge and its differentiation by age, gender and ethnicity in southwestern Niger. Human Ecology 36(6):881-889. dx.doi.org/10.1007/s10745-0089200-7

Besairie, H. \& H. Collignon. 1972. Géologie de Madagascar I, les terrains sédimentaires. Annales Géologiques de Madagascar 35:1-463.

Busch, J., R. Dave, L. Hannah, A. Cameron, A. Rasolohery, P. Roehrdanz \& G. Schatz. 2012. Climate change and the cost of conserving species in Madagascar. Conservation Biology 26(3):408-419. dx.doi.org/10.1111/j.15231739.2012.01838.x

Bussmann, R.W. \& A. Glenn. 2010. Medicinal plants used in Northern Peru for reproductive problems and female health. Journal of Ethnobiology and Ethnomedicine 6:30. dx.doi.org/10.1186/1746-4269-6-30

Byg, A. \& H. Balslev. 2001. Traditional knowledge of Dypsis fibrosa (Arecaceae) in eastern Madagascar. Economic Botany 55(2):263-275. dx.doi.org/10.1007/BF02864564

Callmander, M.W., P.B. Phillipson, G.E. Schatz, S. Andriambololonera, M. Rabarimanarivo, N. Rakotonirina, J. Raharimampionona, C. Chatelain, L. Gautier \& P.P. Lowry II. 2013. The endemic and non-endemic vascular flora of Madagascar updated. Pp. 317-321 in Proceedings of the XIXth AETFAT Congress: 26-30 April 2010, Madagascar. Edited by N. Beau, S. Dessein \& E. Robbrecht. National Botanic Garden of Belgium, Scripta Botanica Belgica, Meise, Flemish Brabant, Belgium.

Camou-Guerrero A., V. Reyes-García, M. Martínez-Ramos \& A. Casas. 2008. Knowledge and use value of plant species in a Rarámuri community: A gender perspective for conservation. Human Ecology 36(2):259-272. dx.doi. org/10.1007/s10745-007-9152-3

Cunningham, A.B. 1996. Peuples, Parc et Plantes: Recommandations pour les zones à usages multiples et les alternatives de développement autour du parc national de Bwindi Impénétrable, Ouganda. Document de Travail Peuples et Plantes no 4. United Nations Educational, Scientific and Cultural Organization (UNESCO), Paris, France. http://unesdoc.unesco.org/images/0010/001091/109173f. pdf
Faramalala, M.H. \& C. Rajeriarison. 1999. Nomenclature des Formations Végétales de Madagascar. Association Nationale de Gestion des Aires Protegees (ANGAP), Antananarivo, Madagascar.

Gade, D.W. 1996. Deforestation and its effects in highland Madagascar. Mountain Research and Development 16(2):101-116. www.jstor.org/stable/3674005

Gallegos, C.M. 1997. Madagascar: Unrealized potential in natural resources. Journal of Forestry 95(2):10-15.

Goodman, S.M. \& B.D. Patterson. 1997. Natural Change and Human Impact in Madagascar. Smithsonian Institution Press, Washington, D.C., U.S.A.

Green, G.M. \& R.W. Sussman. 1990. Deforestation history of the eastern rainforests of Madagascar from satellite images. Science 248(4952):212-215. dx.doi.org/10.1126/ science. 248.4952 .212

IUCN Red List. 2013. Guidelines for Using the IUCN Red List Categories and Criteria. Version 10. Prepared by the Standards and Petitions Subcommittee of the IUCN Species Survival Commission. www.iucnredlist.org/documents/RedListGuidelines.pdf

Lance, K., C. Kremen \& I. Raymond. 1994. Extraction of Forest Products Quantitative of Park and Buffer Zone and Long-Term Monitoring. Report to Park Delimitation Unit, Wildlife Conservation Society/PCDIM, Antananarivo, Madagascar.

Lyon, L.M. \& L.H. Hardesty. 2005. Traditional healing in the contemporary life of the Antanosy people of Madagascar. Ethnobotany Research \& Applications 3:287-294. http://journals.sfu.ca/era/index.php/era/article/view/80

MadCat (Madagascar Catalogue). 2012. Catalogue of the Vascular Plants of Madagascar. Missouri Botanical Garden, Saint Louis, Missouri, U.S.A., and Missouri Botanical Garden - Madagascar Research and Conservation Program, Antananarivo, Madagascar. www.efloras.org/Madagascar Accessed September 2012.

Martin, G.J. 1995. Ethnobotany: A methods manual. Chapman and Hill, London, U.K.

Mittermeier, R.A., I. Tattersall, W.R. Konstant, D.M. Meyers \& R.B. Mast. 1994. Lemurs of Madagascar. Tropical field guide series. Conservation International, Washington, D.C., U.S.A.

Morat, P. 1973. Les Savanes du Sud-Ouest de Madagascar. Mémoires ORSTOM $n^{\circ} 68$. Office de la Recherché Scientifique et Technique Outre-Mer (ORSTOM), Paris, France. 
Novy, J.W. 1997. Medicinal plants of the eastern region of Madagascar. Journal of Ethnopharmacology 55(2):119126.

Quansah, N. 1988. Ethnomedicine in the Maroantsetra region of Madagascar. Economic Botany 42(3):370-375. dx.doi.org/10.1007/BF02860161

Rajerison, R. 1999. Impacts de I'Utilisation de Quelques Plantes Médicinales dans la Région de Manongarivo. Mémoire de DEA, option Ecologie Végétale, Faculté des Sciences, Université d'Antananarivo, Anatananarivo, Madagascar.

Rakotoarivelo, N.H., A.A. Razanatsima, F. Rakotoarivony, A. Kuhlman, S. Andriambololonera, R.H. Ramanajanahary, A. Randrianasolo \& R.W. Bussmann. 2013. Guide des Plantes d'Ambalabe. Volume 1. Missouri Botanical Garden Press, Saint Louis, Missouri, U.S.A.

Rakotoarivony, F. \& L. Rasoavity. 2007. Conservation Communautaire de la Forêt de Vohibe-Ambalabe Vatomandry, Région. Atsinanana, Madagascar.

Randrianarivelojosia, M., V.T. Rasidimanana, H. Rabarison, P.K. Cheplogoi, M. Ratsimbason, D.A. Mulholland \& P. Mauclère. 2003. Plants traditionally prescribed to treat tazo (malaria) in the eastern region of Madagascar. Malaria Journal 2:25. dx.doi.org/10.1186/1475-2875-2-25

Raveloson, T.F. 2014. The Role of the Women in the Traditional Health-Care System of Ambalabe, Madagascar. MS thesis, University of Antananarivo, Ecologie Végétale, Faculté des Sciences, Antananarivo, Madagascar.

Razafindraibe M., A.R. Kuhlman, H. Rabarison, V. Rakotoarimanana, C. Rajeriarison, N. Rakotoarivelo, T. Randrianarivony, F. Rakotoarivony, R. Ludovic, A. Randrianasolo \& R.W. Bussmann. 2013. Medicinal plants used by women from Agnalazaha littoral forest (Southeastern Madagascar). Journal of Ethnobiology and Ethnomedicine 9:73. dx.doi.org/10.1186/1746-4269-9-73

Shangali C.F., I.J.E. Zilihona, P.L.P. Mwang'ingo \& M. Nummelin. 2008. Use of medicinal plants in the eastern Arc Mountains with special reference to the Hehe ethnic group in the Udzungwa Mountains, Tanzania. Journal of East African Natural History 97(2):225-254. dx.doi. org/10.2982/0012-8317-97.2.225

Wayland, C. 2011. Gendering local knowledge: Medicinal plant use and primary health care in the Amazon. Medical Anthropology Quarterly 15(2):171-188. dx.doi. org/10.1525/maq.2001.15.2.171 